\title{
Bubbles apparent magnitudes Messier objects
}

\author{
P.M. Mazurkin ${ }^{1}$ \\ 1. Volga State University of Technology, \\ Yoshkar-Ola, 424000, Republic of Mari El, Russian Federation
}

\begin{abstract}
On the example of the star objects given in the catalog of Mesye, mutual factorial communications between two parameters of the measured objects are considered: in distance and visible star size. The way of transformation of the initial table of data in tabular model is shown. The method of identification of steady regularities is in detail stated in the factorial analysis of these two parameters of objects. Possibility of use of fundamental physical constants on the example of Archimedes's number or space number is shown when transforming statistical model to steady regularity in the form of an asymmetric wavelet signal. It is proved that the measured values of distance and visible star size have a big error. However under the terms of adequacy the revealed models were strong with correlation coefficient more than 0.7 . For the full factorial analysis more reliable tabular models are necessary. Therefore, the proof of the two bubbles apparent magnitudes in the article is a hypothesis, based on the statistical regularities of changes in the distance depending on the apparent magnitude. Apparently, the shape of the bubble long distance indicates the direction of the dark flow.
\end{abstract}

Key words: catalog of Mesye, distance, visible star size, regularity, bubbles.

\section{Introduction}

By data [1] from a site http://astromaniya.at.ua/ took the table of basic data on 110 objects of the catalog of Mesye (pictures of objects from the catalog of Messier here are placed).

From this table for statistical modeling [2-7] accepted two columns with quantitatively presented two parameters of space shining objects:

$r$ - distance, $10^{3}$ light-year (ly);

$m$ - the visible star size, that is the dimensionless numerical value showing brightness of object and characterizing a stream of energy from shining object per unit area.

Changes in the massif of basic data are necessary for possibility of modeling.

After clarification obtained so-called tabular model. Thus, the tabular model is convenient for input in the

Corresponding author: P.M. Mazurkin, Doctor of Engineering Science, professor, Academician of RANS, member of EANS, research fields: integer primes, distribution, statistical model, identification. E-mail: kaf_po@mail.ru. program Curve Expert [8] environment a form of submission of quantitative statistical data.

\section{Tabular model}

It is brought in table 1. Here except two columns with parameters $r$ and $m$ two more columns with numbers and names of objects also are given. At number of parameters more than two in the general tabular model can be crossed out sections in some cages (that complicates preparation of files for the program CurveExpert environment).

However in tabular model with two columns crossed out sections or lack of numerical values in cages aren't allowed. Therefore we removed the lines M40 and M73. Then remain the 108 th object.

The factorial analysis of tabular model assumes a binary combination of all considered factors. In this article of the relation of factors from themselves (rank distributions) we don't consider.

Therefore remain only $2^{2}-2=2$ binary relations in the form of required regularities of type $m=f(r)$ and $r=f(m)$. 
Table 1 Catalog of objects of Messier

\begin{tabular}{|c|c|c|c|c|c|c|c|}
\hline № & $N$ & $r, 10^{3}$ & $m$ & № & & $r, 10^{3}$ & $m$ \\
\hline प1 & NGC & 6.3 & 8.0 & $\overline{456}$ & NGC 6779 & 32 & 9.5 \\
\hline $\mathrm{M} 2$ & \begin{tabular}{|l|} 
NGC 7089 \\
\end{tabular} & 36 & 7.5 & 457 & NGC 6720 & 4.1 & \\
\hline$\overline{43}$ & NGC 5272 & 31 & 7.0 & & & 500 & \\
\hline$\overline{14}$ & 121 & 7 & 7.5 & & & 0000 & \\
\hline $1 J$ & NGC 5904 & 23 & 7.0 & 160 & & 0000 & 10. \\
\hline M6 & \begin{tabular}{|l|} 
NGC 6405 \\
\end{tabular} & 2 & 4.5 & 161 & 4303 & 0000 & 10. \\
\hline M7 & NGC 6475 & 1 & 3.5 & 462 & NGC 6266 & 22 & 8.0 \\
\hline$\overline{18}$ & NG & 6.5 & 5.0 & & & $\overline{7} \times 1$ & \\
\hline 10 & & 26 & \begin{tabular}{|l|l|}
90 \\
\end{tabular} & $\overline{164}$ & & & \\
\hline & & 13 & 7.5 & 165 & & & 10. \\
\hline 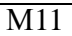 & GC 6705 & 6 & 7.0 & 166 & 3627 & 5000 & 10. \\
\hline 12 & GC 6218 & 18 & 8.0 & 467 & NGC 2682 & 2.25 & 7.5 \\
\hline 1 & & 22 & 7.0 & & & 32 & \\
\hline & & 27 & 9.5 & & & 2 & \\
\hline & & 33 & 1.5 & 170 & & & \\
\hline & 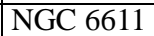 & 7 & 0.5 & 171 & & 12 & 3.5 \\
\hline & FC 6618 & 5 & 7.0 & 172 & & 53 & 10. \\
\hline & & 6 & & & & 35000 & \\
\hline & & 27 & & & & & \\
\hline & & 2.2 & 5.0 & 176 & & & \\
\hline & & 3 & 7.0 & 477 & & 50000 & 0. \\
\hline & $\mathrm{CH}$ & 10 & 6.5 & & & 1.6 & 8.0 \\
\hline & & 4 & & & & 4( & 3.5 \\
\hline & & 10 & 11. & & & & 3 \\
\hline & & & 4 & & & & $3.5-5-3$ \\
\hline & & J & 7 & & & & \\
\hline & & 1.25 & 75 & & & & 0 \\
\hline & & & & & & & 1. \\
\hline 2 & & & & & & & 10 . \\
\hline & & 25 & & & & & 11. \\
\hline & & & 7 & & & & 11 \\
\hline & & & 10.0 & & & & \\
\hline & & & 7.0 & & & & \\
\hline & & & 6.0 & & & & 11 \\
\hline & & & $J$ & & & $\overline{0000}$ & 11. \\
\hline & & & 6 & & & & 7.5 \\
\hline & & & & & & & 6.5 \\
\hline & & & & & & & \\
\hline & & & 5.5 & & & 00 & 1 \\
\hline & & & 5.0 & & & 38000 & 10. \\
\hline & & & 5.0 & & & 2.6 & 12. \\
\hline & & & & & & & 11 \\
\hline & & & & & & & \\
\hline & & 0 & 1.4 & M100 & & 00 & 10 \\
\hline & & .4 & 6.5 & & & 4000 & 8.5 \\
\hline & & 1.6 & 4.5 & & 866 & 0000 & 10. \\
\hline & & & & & & & \\
\hline & & (200 & & & & & \\
\hline & & 3 & 770 & & & & \\
\hline & 5194 & 7000 & 8.0 & & 258 & 25000 & 95 \\
\hline & 654 & 7 & 8.0 & 77 & 71 & 20 & 10. \\
\hline & & $\pi$ & 8.5 & & & & \\
\hline & & 0. & $\cdots$ & & & 00 & \\
\hline & & & & & & & \\
\hline
\end{tabular}

Scope of parameters is equal: the distance changes from $0.3 \times 10^{3}$ ly to $60000 \times 10^{3}$ ly (or to $60 \mathrm{Mly}$ ); visible star size changes from 3.5 to 12.0 . The more scope, the informative required regularities. The standard form [2-7] of the identified polynomial equation in the form of asymmetric wavelet signal is thus applied.

\section{Influence of distance on size $m$}

It appeared, visible star size depends on distance (Fig. 3) on Weibull distribution

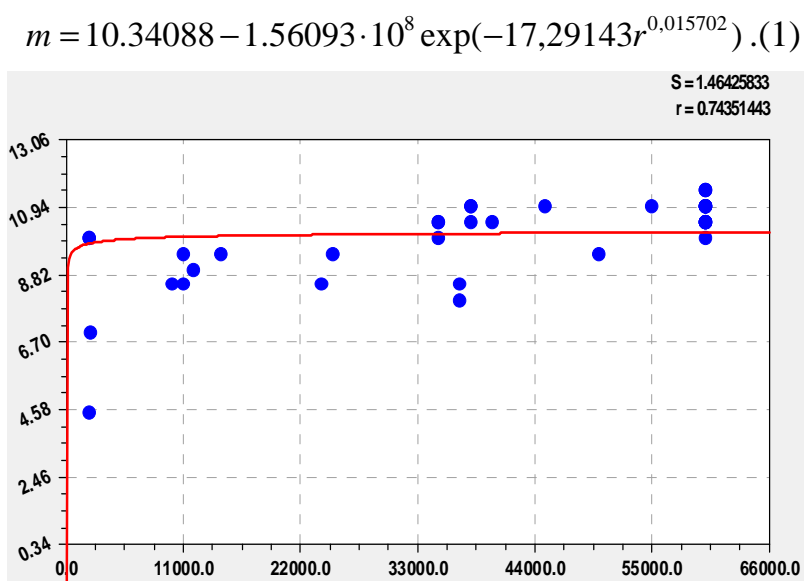

Fig. 1 The determined model of influence of distance at a visible star size.

The coefficient of correlation of a formula (1) is equal 0.7435 (in the right top corner of the schedule). It more admissible level 0.7 therefore the formula (1) belongs to strong binary communications.

The remains after a formula (1), that is its absolute error, allowed to receive infinite wavelet [2-6] (Fig. 2).

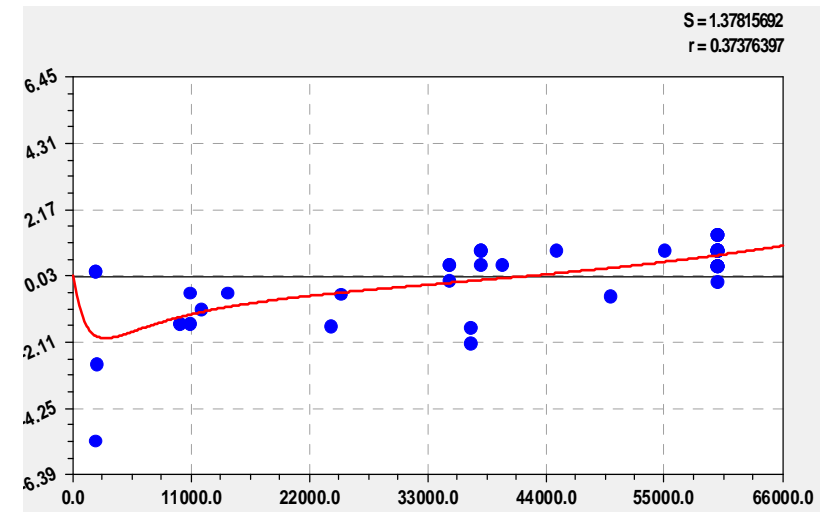

Fig. 2. The wave schedule additional to a formula (1) influence of distance at a visible star size

The schedule of fluctuation is identified by a formula

$$
m_{2}=A \cos (\pi r / p-1,60950),
$$

$$
A=-1.83020 \exp \left(2.56288 \cdot 10^{-5} r^{1.01017}\right) \text {, }
$$




$$
p=2787.4984+0.91650 r^{1.00037},
$$

where $A$ - half of amplitude of fluctuation of star size, $p$ - wave half-cycle, $10^{3} l y$.

After association of two formulas we receive:

$$
m=m_{1}+m_{2},
$$

$$
\begin{aligned}
& m_{1}=9.83218-1.49173 \cdot 10^{8} \exp \left(-17.33746 r^{0.020294}\right), \\
& m_{2}=A \cos (\pi r / p-1.57699), \\
& A=-1.62503 \exp \left(2.41467 \cdot 10^{-5} r^{1.00693}\right), \\
& p=2634.417+0.91213 r^{0.99978} .
\end{aligned}
$$

We notice that wave shift in a formula (2) was equal $1.6095010^{3} \mathrm{ly}$, and in expression (3) it became 1.57699 that is close to value $\pi / 2=1.570795$.

The method of approach of parameters of statistical regularities was shown in article [5] to fundamental physical constants. In a formula (2) such constants become number $\pi$ (Archimedes's number or space number).

Therefore we will replace $1.57699 \rightarrow \pi / 2$ and then instead of the $\cos$ function we will receive the $\sin$ function.

We start the program environment [8] and after identification we receive (Fig. 3) formula

$$
m=m_{1}+m_{2},
$$

$$
\begin{aligned}
& m_{1}=9.83599-1.48990 \cdot 10^{8} \exp \left(-17.33770 r^{0.020334}\right), \\
& m_{2}=A \sin (\pi r / p), \\
& A=-1.63350 \exp \left(2.38778 \cdot 10^{-5} r^{1.00502}\right), \\
& p=2671.4718+0.91268 r^{0.99981} .
\end{aligned}
$$

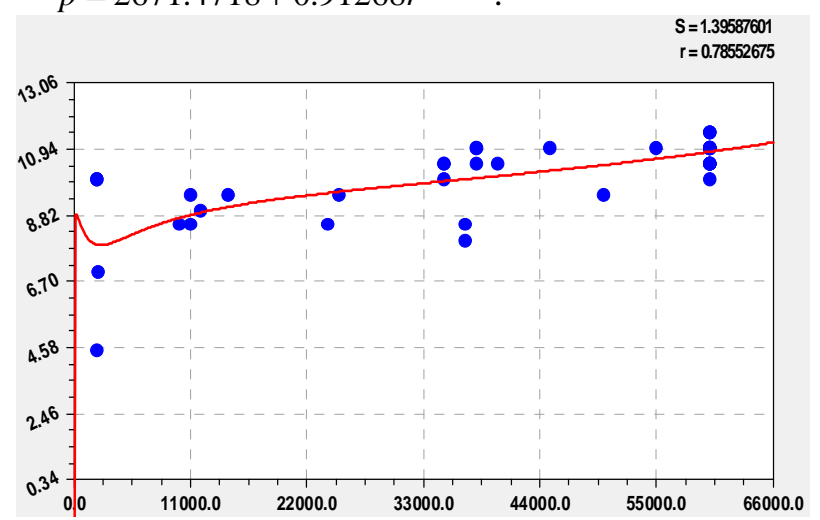

Fig. 3 The schedule of a tripartite formula (4) influences of distance at a visible star size.

The coefficient of correlation grew to 0.7855 . From the schedule values of ordinate abnormally located concerning abscissa axis are visible.
For example, at $r=220010^{3}$ ly there are three points of visible star size (in table 1 they are allocated): 4.5 7.0 and 10.0. Such wide spacing of values of parameters doesn't give the chance to apply a method of physical constants [5].

\section{Reduction of statistical selection}

From table 1 we clean far objects (tab. 2) and we leave those lines, which less $8310^{3} \mathrm{ly}$.

Table 2 The reduced catalog of objects of Mesye

\begin{tabular}{|c|c|c|c|c|c|c|c|}
\hline № & № NGC & $\begin{array}{c}\text { ly } \\
\text { M1 }\end{array}$ & $m$ & № & № NGC & $\begin{array}{c}r, 10^{3} \\
\text { ly }\end{array}$ & $m$ \\
\hline M2 & NGC 7052 & 6.3 & 8.0 & M41 & NGC 2287 & 2.4 & 5.0 \\
\hline M3 & NGC 5272 & 36 & 7.5 & M42 & NGC 1976 & 1.6 & 5.0 \\
\hline M4 & NGC 6121 & 7 & 7.5 & M44 & NGC 2632 & 0.5 & 4.0 \\
\hline M5 & NGC 5904 & 23 & 7.0 & M46 & NGC 2437 & 5.4 & 1.4 \\
\hline M6 & NGC 6405 & 2 & 4.5 & M48 & NGC 2548 & 1.5 & 5.5 \\
\hline M7 & NGC 6475 & 1 & 3.5 & M50 & NGC 2323 & 3 & 7.0 \\
\hline M8 & NGC 6523 & 6.5 & 5.0 & M53 & NGC 5024 & 56 & 8.5 \\
\hline M9 & NGC 6333 & 26 & 9.0 & M54 & NGC 6715 & 83 & 8.5 \\
\hline M10 & NGC 6254 & 13 & 7.5 & M55 & NGC 6809 & 17 & 7.0 \\
\hline M13 & NGC 6205 & 22 & 7.0 & M56 & NGC 6779 & 32 & 9.5 \\
\hline M14 & NGC 6402 & 27 & 9.5 & M57 & NGC 6720 & 4.1 & 9.5 \\
\hline M15 & NGC 7078 & 33 & 7.5 & M62 & NGC 6266 & 22 & 8.0 \\
\hline M17 & NGC 6618 & 5 & 7.0 & M67 & NGC 2682 & 2.25 & 7.5 \\
\hline M19 & NGC 6273 & 27 & 8.5 & M68 & NGC 4590 & 32 & 9.0 \\
\hline M20 & NGC 6514 & 2.2 & 5.0 & M69 & NGC 6637 & 25 & 9.0 \\
\hline M21 & NGC 6531 & 3 & 7.0 & M70 & NGC 6681 & 28 & 9.0 \\
\hline M23 & NGC 6494 & 4.5 & 6.0 & M71 & NGC 6838 & 12 & 8.5 \\
\hline M25 & NGC 4725 & 2 & 4.9 & M72 & NGC 6981 & 53 & 10.0 \\
\hline M26 & NGC 6694 & 5 & 9.5 & M75 & NGC 6864 & 58 & 9.5 \\
\hline M27 & NGC 6853 & 1.25 & 7.5 & M76 & NGC 650 & 3.4 & 12.0 \\
\hline M29 & NGC 6913 & 7.2 & 9.0 & M78 & NGC 2068 & 1.6 & 8.0 \\
\hline M30 & NGC 7099 & 25 & 8.5 & M79 & NGC 1904 & 40 & 8.5 \\
\hline M34 & NGC 1039 & 1.4 & 6.0 & M80 & NGC 6093 & 27 & 8.5 \\
\hline M35 & NGC 2168 & 2.8 & 5.5 & M92 & NGC 6341 & 26 & 7.5 \\
\hline M36 & NGC 1960 & 4.1 & 6.5 & M93 & NGC 2447 & 4.5 & 6.5 \\
\hline M37 & NGC 2099 & 4.6 & 6.0 & M97 & NGC 3587 & 2.6 & 12.0 \\
\hline M38 & NGC 1912 & 4.2 & 7.0 & M103 & NGC 581 & 8 & 7.0 \\
\hline M39 & NGC 7092 & 0.3 & 5.5 & M107 & NGC 6171 & 20 & 10.0 \\
\hline
\end{tabular}

Statistical modeling showed that it is necessary to exclude and couples: $1.64 .5 ; 1.67 .5 ; 67.0 ; 6$ 8.0; 7 $6.5 ; 78.0 ; 106.5 ; 10$ 11.5; 18 8.0; 18 8.5.

After identification of table 2 it was received (Fig. 4) finite-dimensional wavelet in the form of a formula

$$
\begin{gathered}
m_{3}=A_{2} \cos \left(\pi r / p_{2}+2.36693\right), \\
A_{2}=22.76948 r^{0.64385} \exp \left(-2.81627 r^{0.20783}\right), \\
p_{2}=0.25207-0.014555 r^{0.48901} .
\end{gathered}
$$

The coefficient of correlation asymmetric wavelet with variables amplitude and the period of fluctuation is equal 0.5078 . 
In a formula (2) the period growth (calm of fluctuation) was observed, and in a formula (5) there is an increase of a lonely wave.

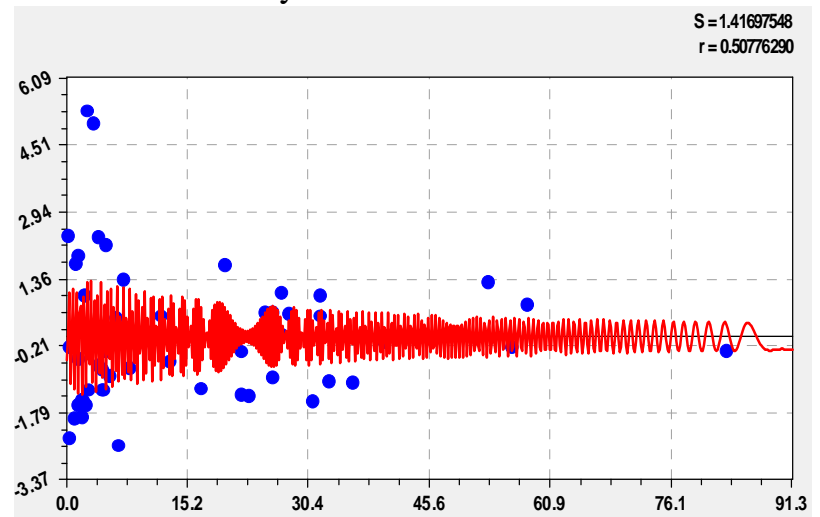

Fig. 4 Schedule wavelet (5) wave influences of distance on visible star size.

Further we will unite together four components.

\section{Association of regularities}

After repeated identification it was received (Fig. 5) four-membered model (Weibull law has two components) look

$$
m=m_{1}+m_{2}+m_{3},
$$

$$
\begin{aligned}
& m_{1}=9.87454-1.48990 \cdot 10^{8} \exp \left(-17.34245 r^{0.020334}\right), \\
& m_{2}=A_{1} \sin \left(\pi r / p_{1}\right), \\
& A_{1}=-1.64029 \exp \left(2.38710 \cdot 10^{-5} r^{1.00502}\right), \\
& p_{1}=2724.9779+0.91335 r^{0.99981}, \\
& m_{3}=A_{2} \cos \left(\pi r / p_{2}+2.35025\right), \\
& A_{2}=21.46863 r^{0.64385} \exp \left(-2.89017 r^{0.20783}\right), \\
& p_{2}=0.25219-0.014586 r^{0.48901} .
\end{aligned}
$$

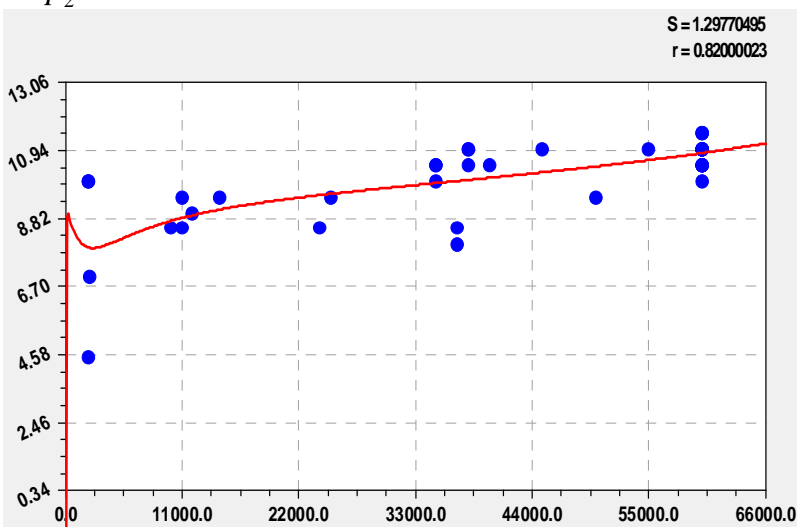

Fig. 5 The schedule of the general model (6) of influence of distance at a visible star size.
Correlation coefficient as a measure of narrowness of factorial communication, reached 0.8200 . From a formula (6) under a condition $r=0$ we will receive expression

$$
\begin{gathered}
m(r=0)=m_{1}+m_{2}, \\
m_{1}=9.87454-1.48990 \cdot 10^{8}, \quad m_{2}=A_{1} \sin \left(\pi r / p_{1}\right), \\
A_{1}=-1.64029, \quad p_{1}=2724.977910^{3} \mathrm{ly} .
\end{gathered}
$$

Thus, at zero distance is negative value of the indicator $m$, approximately equal $-1.5 \mathrm{e} 8$.

To obtain adequate models required the measured data at distances less $0.3 \times 10^{3}$ ly.

\section{Influence $m$ on distance $r$}

Inverse function shows that physically the distance to object of Mesye depends on visible star size.

This hypothesis is confirmed by the binomial determined regularity (Fig. 6) look

$$
r=1231.1022 \exp (0.018076 m)+
$$

$$
+2.55496 \cdot 10^{-122} m^{200.92867} \exp \left(-14.94167 m^{1.06255}\right) \text {. }
$$

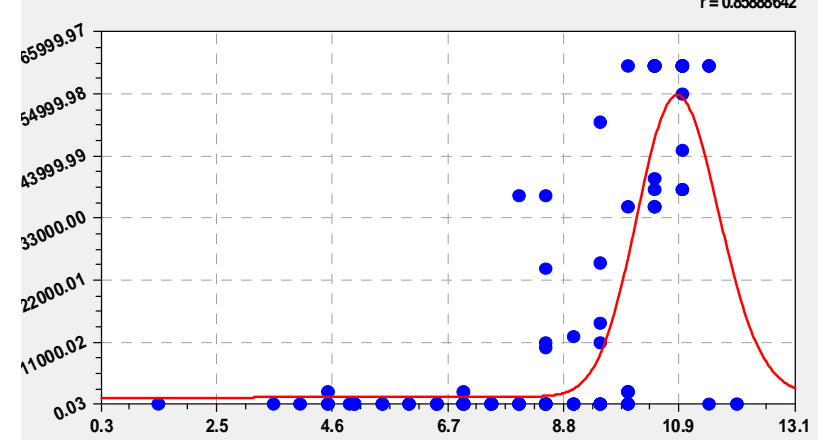

Fig. 6 The return influence of visible star size on distance to object from the catalog of Messier.

The first component (8) under Laplace's law (in mathematics) or Mandelbrot (in physics) exponential growth shows increase in distance to objects with increase of visible star size. And the second component indicates stressful excitement of objects from the catalog of Mesye: in the range of visible star size 8.0-14.0 there is a distance change under the biotechnical law [2-7].

Thus, at change of visible star size to 8.0 and after 14.0 distance to objects doesn't exceed $220010^{3} \mathrm{ly}$. Thus at most a distances, much more bigger $6000010^{3}$ 
$l y$, apparently, it is expected at optimum visible star size $m^{*}=11$.

This suggests that the distance to the object is unknown until the physical sense.

From the remains after (8) in figure 7 it is visible that in the range of visible star size from 8 to 14 it is necessary to increase the accuracy of measurements of distance.

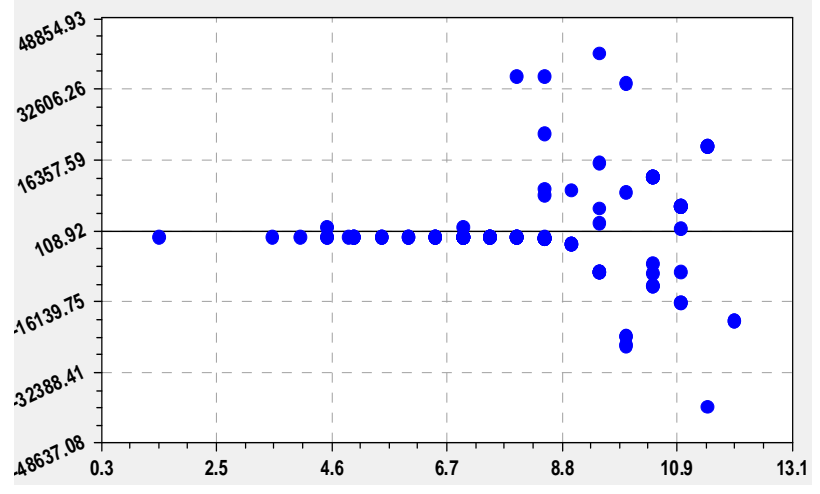

Fig. 7 The remains after model (8) of influence of visible star size on distance to object of Messier.

To understand physical sense, the set of the new tabular models received from different catalogs of star objects is necessary. And basic data for statistical modeling by an identification method with a small error of measurements of parameters of objects are necessary.

\section{About bladder apparent magnitude}

The first component of formula (8)

$$
r_{1}=1231.1022 \exp (0.018076 m)
$$

It shows a slight increase in border puzy convent depending on the apparent magnitude.

The figure 6 shows a second bubble is formed in a narrow range of variation of apparent magnitude.

From the remnants of Figure 7 shows that when reduced-Research Institute of measurement error of both parameters can be obtained by further wave dependency.

\section{On the dark stream}

Apparently, the second component

$$
r_{2}=2.55496 \cdot 10^{-122} m^{200.92867} \exp \left(-14.94167 m^{1.06255}\right)(8 \mathrm{~b})
$$

It shows a family of distant objects.

If a table model were the coordinates of each object, apparently, one would determine the direction of the axis of the dark currents.

But this requires other spreadsheet models.

\section{Conclusions}

The method of identification of [2-7] asymmetric wavelet signals allows to reveal strong regularities and on other tabular models made on the basis of numerous astronomical measurements. Thus the number of parameters with numerical values in tabular model can be much more than two.

On the basis of the catalog of objects of Mesye, for an identification example, it was succeeded to allocate two parameters with numerical values: $r$ - distance $10^{3} \mathrm{ly} ; m$ - visible star size.

It was shown that the factorial analysis on a set of parameters of objects from which the greatest interest unknown while present to science the return regularities, in our example of $r=f(m)$ is possible.

Thus need for allocation of structural formulas at detection of regularities disappears. All parameters of star objects become equivalent therefore all binary relations can be considered.

However existing data files by results of measurements include not only quantitative values of parameters of star objects.

It is a lot of text explanations and any codes which don't give in to mathematical processing. It very much complicates statistical modeling. Tables of data at least as the Mesye [1] catalog are necessary to us.

Therefore we address to readers: provide to us on e-mail kaf_po@mail.ru from the ocean of existing statistical information on star objects of the table as the catalog of Mesye (thus coordinates at objects also have to be distributed on three columns).

\section{References}

[1] Catalog of objects of Mesye: http://astromaniya.at.ua/Catalog_Mesye/Catalog_Mesye. $\underline{\mathrm{html}}$ 
[2] P.M. Mazurkin, "Asymmetric Wavelet Signal of Gravitational Waves." Applied Mathematics and Physics, vol. 2, no. 4 (2014): 128-134. doi: 10.12691/amp-2-4-2.

[3] P.M. Mazurkin, "Wavelet Analysis of a Number of Prime Numbers." American Journal of Numerical Analysis, vol. 2, no. 2 (2014): 29-34. doi: 10.12691/ajna-2-2-1.

[4] P.M. Mazurkin. Patterns of primes. Germany : Palmarium Academic Publishing, 2012. 280 p.

[5] P.M. Mazurkin, "Stable Laws and the Number of Ordinary." Applied Mathematics and Physics, vol. 2, no. 2 (2014): 27-32. doi: 10.12691/amp-2-2-1.
[6] P.M. Mazurkin, "Identification of statistical stable patterns." SCIENCE AND WORLD. International scientific journal, № 3 (3), 2013. p.28-33.

[7] P.M. Mazurkin, A.S. Filonov, "Mathematical modeling. Identification univariate statistical regularities: tutorial." Yoshkar-Ola, Mari State Technical University, 2006. 292 p.

[8] “CurveExpert", URL: http://www.curveexpert.net/. 